\title{
A case of early localized scleroderma that was manifested as a port-wine stain
}

\author{
Qinqin Meng, Meiliang Guo, Dingfen Yuan, Hui Deng
}

Department of Dermatology, Shanghai Jiao Tong University Affiliated Sixth People's Hospital, Shanghai, China

Adv Dermatol Allergol 2021; XXXVIII (4): 701-702

DOI: https://doi.org/10.5114/ada.2021.108897

Port-wine stain (PWS) and localized scleroderma (LS) are easy to be identified in the late stage of diseases. However, several reports of early morphea present an early underrecognized inflammation that resembles PWS. This case was presented to highlight the early diagnosis of LS, which manifests as PWS. Misdiagnosis can delay the diagnosis of scleroderma and cause some other serious complications.

A 12-year-old girl presented with an erythematous patch along the right forehead associated with slight redness of her right inner eye for 1 year (Figure 1). The dermoscopic examination revealed telangiectasias. She denied trauma history and was otherwise healthy. A clinical diagnosis of PWS was made and she was treated with a pulsed dye laser (PDL). After a treatment session for about 6 months, erythema totally faded away, along with mild atrophy and white spots on the right forehead near the hair. The skin lesions hardened. The diagnosis of LS was suspected, and a skin biopsy was conducted to confirm the diagnosis.

The skin biopsy of the white spots on the right forehead near the hair revealed epidermal atrophy, thickened collagen bundles along with atrophic hair follicles and sebaceous glands, and perivascular lymphocytic infiltrates (Figure 2).

After the diagnosis of LS was confirmed, the patient was advised to have her possible complications checked on a regular basis at the hospital. She was subsequently treated with $12 \mathrm{mg}$ Centella triterpenes tablets three times a day and application of Centella triterpenes cream to the white spots on the right forehead near the hair three times a day for 2 years, and did not have any obvious complications.

LS is characterized by indurated plaques. The cause of the disease can be explained by several hypotheses, such as genetic background; environmental factors; radiation; medications; infection with Borrelia or cytomegalovirus; autoimmunity with positive antinuclear antibody
(ANA), anti-single-stranded DNA, anti-histone antibodies, and anti-topoisomerase II; and disturbance of the equilibrium between the collagen synthesis-degradation and microvascular injury $[1,2]$. At the same time, trauma is suspected. Treatment with PDL has not been confirmed for the development of morphea. In the early inflammatory stage of morphea, PDL can decrease erythema by completely removing blood vessels. However, in the case of trauma, PDL may stimulate the inflammatory response, which signifies the recruitment and secretion of inflammatory cells, cytokines, and growth factors. This inciting milieu may be pivotal for initiating abnormal collagen production in fibroblasts, which typical of morphea $[2,3]$.

In a survey, 50 patients were diagnosed with LS. The ratio of female:male was $2.8: 1$, with a median age at disease onset of 5.2 years (range: 0.1-14.4). The type of LS was mostly linear (86\%) or a combination of linear and plaque-type (8\%). The patients were first examined by a doctor after $1.2(0.2-48.7)$ months. Initially, none of the doctors could diagnose the disease correctly, $44 \%$ of doctors did not suggest anything regarding the diagnosis, and $20 \%$ of doctors misdiagnosed atopic dermatitis [4].

Early LS presents with erythematous, hyperpigmented, hypopigmented, violaceous, and telangiectatic lesions, which can make the diagnosis difficult. As LS has normal levels of laboratory tests and a notable lack of anticentromere antibodies and anti-scleroderma-70 antibodies, the characteristic clinical manifestations are crucial for early diagnosis. For histopathological analysis, skin biopsy should be performed if LS is suspected. Six clinical clues for diagnosis include erythema, skin tightness, dyspigmentation (hyper- or hypopigmentation), atrophy (of skin and/or underlying tissue), hair loss (scalp hair, eyebrows, or eyelashes), and Blaschko's lines (linear morphea).

Histopathologically, early lesions may show perivascular lymphocytic infiltration. Over time, the inflamma-

Address for correspondence: Hui Deng, Department of Dermatology, Shanghai Jiao Tong University Affiliated Sixth People's Hospital, 600 Yishan Road, Xuhui District, Shanghai, China, e-mail: hdeng@sjtu.edu.cn Received: 9.10.2019, accepted: 7.12.2019. 


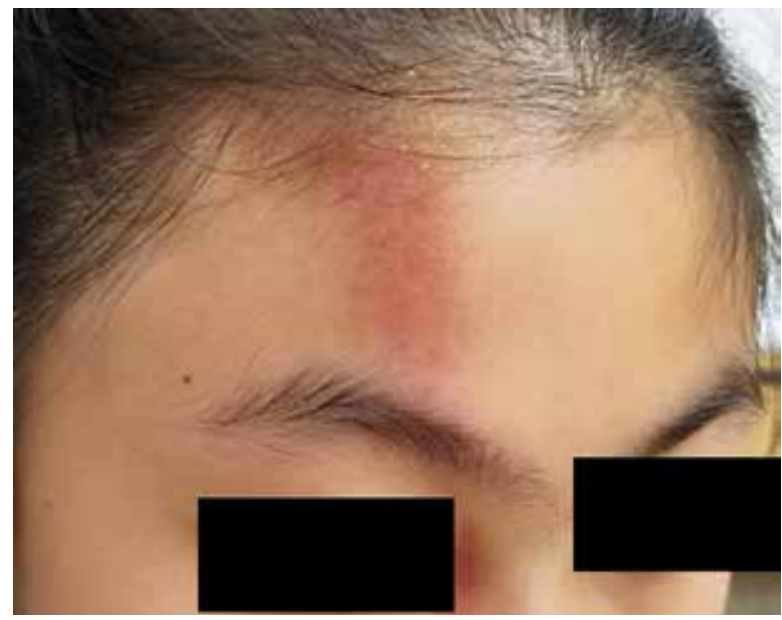

Figure 1. Clinical presentation of an erythematous patch along the right forehead associated with slight redness of her right inner eye

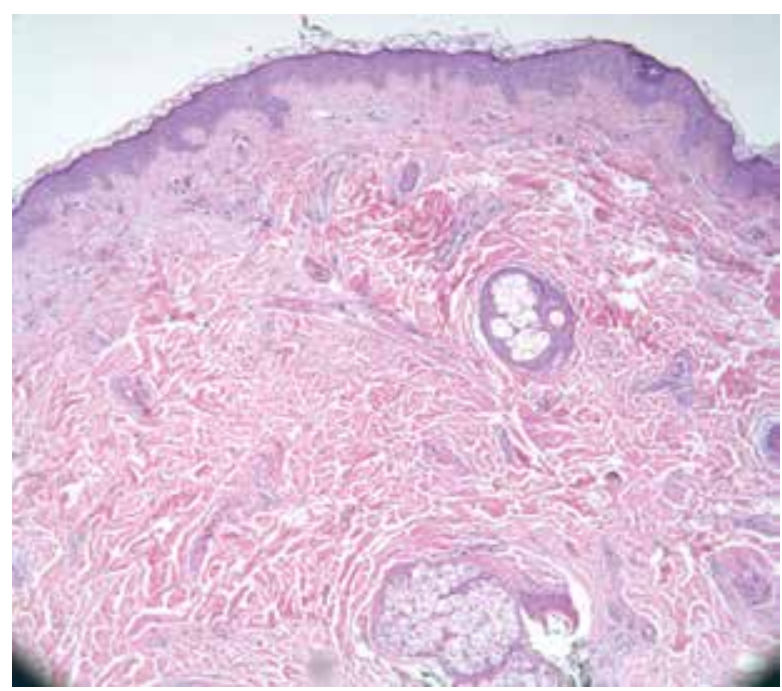

Figure 2. A skin biopsy from the white spots showed epidermal atrophy, thickened collagen bundles along with atrophic hair follicles and sebaceous glands, and perivascular lymphocytic infiltrates. Haematoxylin-eosin staining (100x)

tion dampens and collagen bundles thicken. For some atypical lesions, the diagnosis is considered if perineural inflammation is seen. Inflammation involves T-helper cells and their associated cytokines. Many autoimmune diseases, including scleroderma, are thought to be propagated by an imbalance of Th cell effector lineages and their associated cytokines [5]

Topical $0.1 \%$ tacrolimus cream may be used in patients with morphea, particularly with early inflammatory lesions, even as a first-line treatment [6, 7]. In addition, the effect of low-dose ultraviolet A-1 phototherapy on morphea has also been confirmed in previous studies $[6,8]$.

PWS is a disease caused by capillary malformation, which is manifested as a latent progressive vascular plex- us and is located mostly on the face and neck. Due to its impact on the appearance, PWS can make patients emotionally distressed and affect their quality of life. PWS is characterized by a clear, irregular red or purplish patch, which may fade completely after compression. The incidence of PWS in newborns is $0.3-0.5 \%$, and generally it does not subside. With the lack of treatment, the colour of erythema deepens, the affected area increases, and surface thickening or nodular hyperplasia occurs with advancing age. The pathogenesis of PWS is unclear. One hypothesis indicated that sympathetic nerve loss was responsible for the progressive vascular ectasia [9]. PDL is the standard conventional treatment for PWS.

In conclusion, the present case highlighted that doctors should pay attention to the diagnosis of LS, which may manifest as PWS, especially the skin lesion on the scalp in children. Proper treatment with Centella triterpenes cream and tablets is necessary to prevent the occurrence of complications of scleroderma if LS is confirmed. Either delayed treatment of scleroderma or treatment with PDL can have a possible adverse effect on the disease.

\section{Acknowledgments}

Source of support: National Natural Science Foundation of China (No. 81673054).

\section{Conflict of interest}

The authors declare no conflict of interest.

\section{References}

1. Kacar SD, Ozuguz P, Polat S, et al. Juvenile localized scleroderma with port wine stain: coincidental or possible common pathogenetic association. Indian I Dermatol 2015; 60: 216.

2. Ng SS, Tay YK. Inflammatory morphea mimicking an acquired port-wine stain initially treated with pulsed-dye laser. J Cosmet Laser Ther 2015; 17: 277-80.

3. Pickert AJ, Carpentieri D, Price H, et al. Early morphea mimicking acquired port-wine stain. Pediatr Dermatol 2014; 31: 591-4.

4. Weibel L, Laguda B, Atherton D, et al. Misdiagnosis and delay in referral of children with localized scleroderma. Br J Dermatol 2011; 165: 1308-13.

5. Kurzinski K, Torok KS. Cytokine profiles in localized scleroderma and relationship to clinical features. Cytokine 2011; 55: 157-64.

6. Kim HS, Lee JY, Kim HO, et al. En coup de sabre presenting as a port-wine stain initially treated with a pulsed dye laser. J Dermatol 2011; 38: 209-10.

7. Stefanaki C, Stefanaki K, Kontochristopoulos G, et al. Topical tacrolimus $0.1 \%$ ointment in the treatment of localized scleroderma. An open label clinical and histological study. J Dermatol 2008; 35: 712-8.

8. Gruss CJ, Von Kobyletzki G, Behrens-Williams SC, et al. Effects of low dose ultraviolet A-1 phototherapy on morphea. Photodermatol Photoimmunol Photomed 2001; 17: 149-55.

9. Rosen S, Smoller BR. Port-wine stains: a new hypothesis. J Am Acad Dermatol 1987; 17: 164-6. 